\title{
Virtual Assistant App for Disabled People
}

\author{
Madhu H. S., Nithin Gowda N. S., Srivatsa ,Yashas Gowda H. M., Ramesh B.
}

\begin{abstract}
Human life is heading towards busy and hectic schedule it becomes necessary to automate the home appliances. The main objective of virtual assistant is controlling, managing and co-ordinating surrounding devices in a comfortable, secure and effectiveway. Some methods can control and handle different types of appliances using unique methodology. Virtual Assistant presents the automated approach of controlling the household devices that could ease the tasks of using the traditional methods. Augmented Reality is a recently developedmethod for the automation of various electrical appliances which is used to allow virtual pop ups on the screen when the camera of the smartphone is pointed towards the object. This pop up enables the user to turn on or off the device by simple touch selection thus improvising the ways of automation in a significant way. This application is built in such a platform where a visually challenged one also has a chance to interact with the system through braille method. The entire system concentrates on simple way of interaction. The best way of interaction is through Android Application. Developed app will not only be helpful for disabled persons but also provides areliable and well-mannered platform for each and every individual that helps in saving energy. Face recognition plays a vital role in providing security for the home owner by providing information about the people present at their door-step.
\end{abstract}

Keywords - Virtual assistant, Android application, Augmented Reality $(A R)$, Face recognition

\section{INTRODUCTION}

A use friendly system requires proper way of organizing the components that meet the requirement of the user. The general goal of the virtual assistant is to make use of network technology to combinedifferent devices, appliances and services found in our surrounding so that the entire domestic living space can be controlled remotely. Virtual assistant is a very promising area for assistance. Its main benefit is that increased comfort, greater safety and security are met, along with allowing significant savings of energy. It also offers powerful streams for helping and supporting the special needs of people with disabilities. Braille is a system of touch and sense based reading and writing method which helps blind, in which raised dots denotes the letters of the alphabet. It allows blinds to read, write, and communicate in absence of their vision. Represented with a sequence of raised bumps that correlate to alphabets, numeric, and punctuation marks are joined together to form words and further words to form sentences, it is read by running the hands over the paper from left to right along each line.

Revised Manuscript Received on December 16, 2019.

Mr. Nithin Gowda N. S., Department of CSE, Malnad College of Engineering, Hassan, (Karnataka) India.

Mr. Yashas Gowda H. M., Department of CSE, Malnad College of Engineering, Hassan, (Karnataka) India.

Dr. Ramesh B. is currently working asProfessor in Department of CSE, Malnad College of Engineering, Hassan (Karnataka) India.
For smartphones raised bumps is represented in a different manner where the way of understanding alphabets remains the same which helps us to develop an interface that can communicate with visually impaired people.

Augmented reality (AR) which is an interactive way of experiencing the real world objects that resides computer generated images. AR uses the concept of image tracking, recognizing, processing and communicating that information to control the applications. Web remote control feature enhances the operation of objects over mobile devices. Mobile devices when encounters an appliance, it generates replica of the object and displays operations that can be carried out with that device. Home automation problem [2] is easily tackled in efficient way.

Face recognition is a biometric technology that goes beyond just detecting human faces in an image or video. It goes a bit further to determine whose face it is. A facial recognition system works by taking an image of a face and predicting whether the face matches another face stored in a database. Therefore, the technology is designed to compare and accurately predict potential matches of the face captured irrespective of facial expression, age, or facial hair. This recognition system is installed at the security camera that will be present at the front door. Once recognized convenient messages or alerts are sent to the owner for their future actions.

Artificial intelligence (AI) is the collection of strong, effective and rigorous programming techniques that studies the nature of intelligence by building programs, and the usage of these concepts are influenceable in solving realworld problems. The growth in the areas of AI has increased drastically over the decade. There exists a number of AI tools that make an automation system more sophisticated. The AI system proposed in this project is capable of controlling home appliances on the instruction of the user. For having AI Android application is built which have provision to sustain all the modules or components in a single interface. This interface needs to be user-friendly, easily understandable and easy to handle. Controlling devices is the major concern, so an Arduino is used which serves as a central system or a controller. Each and every data generated concerns with the proper working of the controller.

\section{RELATED WORK}

Survey was done to check the way of having thoughts by different set of people around the world. Survey conducted by Amrita and Sudhir [1] was predominantly to keep track the potential use of personal assistant. The voice recognition and contextual understanding between the assistant and user were met in sufficient way. This survey led to a conclusive result saying that majority of the people will rely on some technologies around them and make better use of it. 


\section{Virtual Assistant App for Disabled People}

Responding to the real time scenarios must be the job of the assistant. Merging machine learning techniques with the existing system will give birth to new possibilities.

As illustrated by [2] system designed by this particular theory was concerned about the problems faced during the development of home automation techniques. Many approaches was being made but this analysis discriminately says that communication modules like Wi-Fi technology and smart devices can bring a huge difference in the trend. Many research is going on regarding the identification of devices.

Raspberry pi which controls the entire can be implemented in any smart devices. A great deal of work is to be carried out to enhance voice instructions.

Tanveer and his mates [3] built a navigation system to guide blinds for their movement assistance and identifying obstacles in the way of their walk. Brain storming methods helped them to efficiently design a system that resulted in less harm to the blinds. Progress in research signified that braille mode of communication would result in much quicker way of response to their work. It was completely hardware based system where new technologies were not sufficiently utilized. Every people relay on technology and smartphones which paves a way to develop an application which includes braille method of communication. Facenet [4] a unique way of recognizing and identifying faces used many convolutionary methods to increase the percentage of matching ability of the original with the input one through CNN methods and algorithm. This algorithm significantly recognizes multiple faces at a single time without much complications since it stores data about the person. More that $90 \%$ of the images are recognized with the help of training data. Face recognition algorithms can be used to provide security. From [3] braille methods can be implemented with android application along with virtual assistance.

The workflow of this system have multiple components integrated into a single android application which is sufficient enough to carry out the work. Smart AI system which integrates AI assistant, Braille API, AR image recognition, voice recognition which are implemented using IoT is simplified and represented as a block diagram Fig 1.

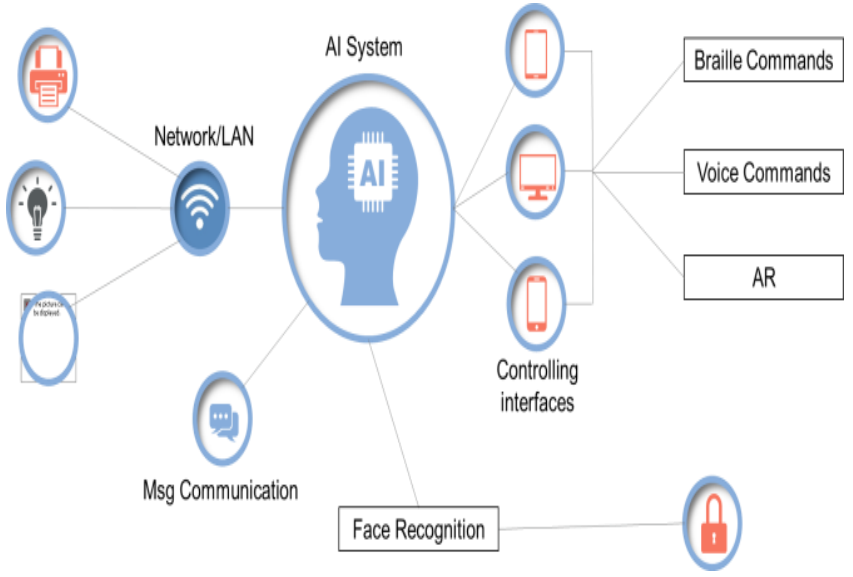

Fig 1. Showing the outer look of the virtual assistant

\section{VIRTUAL ASSISTANTAND RESULTS}

\section{A. Braille implementer}

Braille instructions are implemented in series so that when instructions or commands are sensed via the interface the system should be able to perform the task in reference to the command received. The response taken from the app is the major process which will be processed further by the system as per the predefined instruction set. Braille codes are designed within the UI for user-friendly communication. Here specific commands are assigned with specific tasks so that it will be easier for the user to remember the working technique of the system.

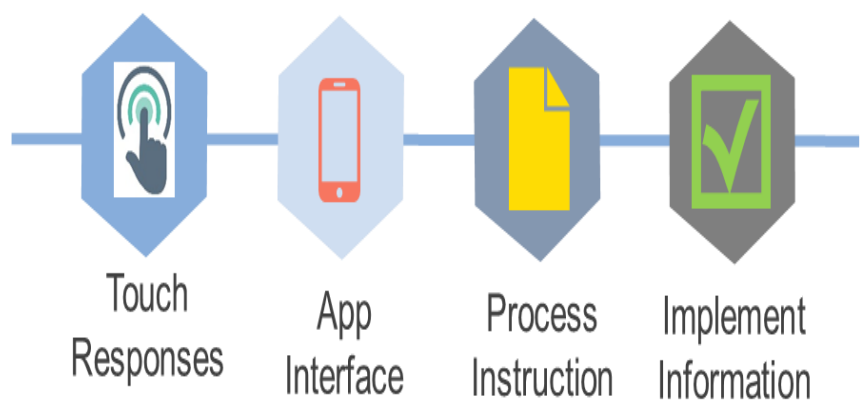

Fig 2. Block diagram showing the implementation of braille method

\section{B. Voice recognition system}

The simplest approach and difficult way of implement is the voice recognizer. This system does the same work as the other voice recognizers does but in a different manner. The voice input from user is recognized and processed so that any commands from the user and that are within the range of system can be manipulated without much work. Learning mechanism are used to train the system so that it results with the desired answers to the user. Multiple layered learning mechanisms is required since the user needs the system to be very interactive with multiple models.

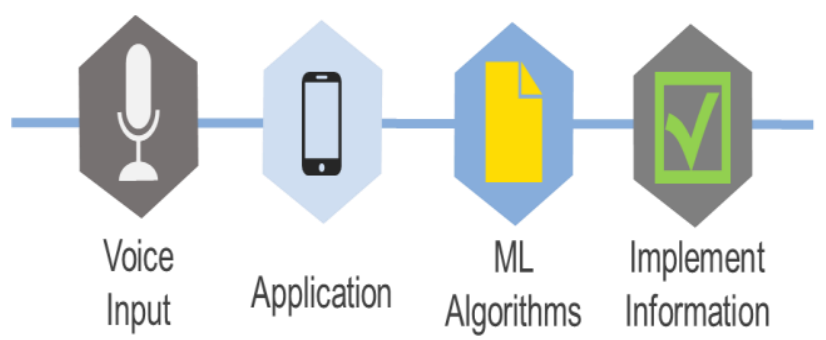

Fig 3. Block diagram showing voice recognition system

\section{Security purpose face identifier}

Face recognition is a field which isused in newest applications. Our system senses the people when they arrive at the door-step and by enabling the face recognition algorithm it reads the faces of the individuals present at the gates and sends needful information like number of people and their identity to the owner of the house.

Published By:

Blue Eyes Intelligence Engineering \& 
If the system does not recognize the individual it send a message to the owner about the people so that security of the house is fulfilled. CNN algorithms are used to detect the face of individuals in much efficient way. A database is used to compare the faces captured by the camera.

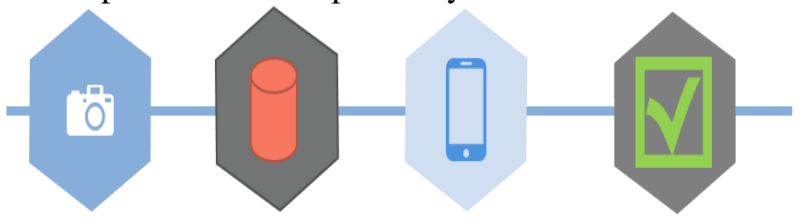

Camera Database App Display information

Fig 4. Face identifier out look

Arduino is the central communicator between multiple components. It receives signals about the status of devices, working ability and controlling ability. A centralized systems structure is represented in Fig 5.

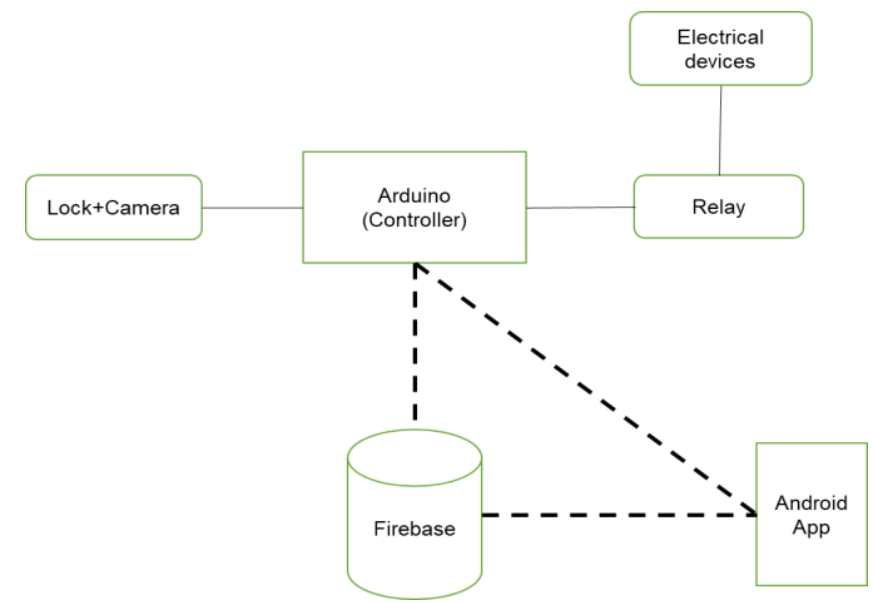

Fig 5. Interconnection between the modules.

\section{RESULTS AND DISCUSSIONS}

Md. Siddiqur Rahman Tanveer and team [3] presented a blind assistive embedded system for tracking path. In this system the blind people can navigate via the information given by the android application. Blind onesare guided through voice replay generated within the application based on the obstacle position sensed by the sensor. Referring to the voice command, a blind person can take necessary actions. Avni Sharma and team [2] used Augmented reality (AR) that allowsvirtual pop-up of image when the camera of the smartphone is positionedinfront of the device, the pop up provides options such as turn on or off the destinated objects work by simple touch selection thus improvising ways of automation. This paper is fully concerned on the reduction of the human power. Amrita S. Tulshan and team [1] used Intelligent Virtual Assistant (IVA) for voice recognition. IVA's usage were documented so that complete information is utilized for calculating the group of individuals who are dependent on this technology. Voice engaged IVAs like Microsoft, Alexa and Google Assistant largelyinfluence on activities carried on continuously in homes. Proposed system is going to be completely different from existing one, above mentioned existing systems will concentrate on particular area.
In [3], system is concentrated only on assisting blind peoples by voice replay and there will be no voice commands are given, but proposed system uses voice recognizer which is more helpful for blind peoples and also this system uses braille method to assist for blind people. [2] the system uses Augmented reality (AR) just to recognize the element when the camera of the smartphone is pointed towards the it, but in proposed system when a smartphone pointed towards the element (ex: fan) the pop up enables options like turn on or off the operation by simple touch selection thus improvising ways of home automation by the same time voice commands also given which helps for blind people to do the work. [1] In this paper they are used virtual assistant to use in homes where only normal peoples can use this system, but in proposed system the virtual assistance mainly maid for the disabled peoples like deaf, dumb or both deaf and dumb, etc. Proposed system which can also be used by the normal peoples. In additional to these features a door step security from [4] is included to make it a better app with fully functional and beneficial units.

\section{EXPECTED OUTCOMES}

Firstly, the main outcome is voice assistant which will be very useful for blind and dumb people to interact with home appliances. Another outcome is converting the braille language to text, which is very important for bling people to understand and communicate and utilize the system in sufficient manner. Next, conversion of images to text formats or voice formats which is very useful for physically challenged people. Controlling of electronic devices in home such fan or bulbs, which will make a physically challenged person life easier. Along with these, creating a smart lock for doors using face recognition system will help them to recognize people who are on the door, and will also help them when they are away from home.

\section{CONCLUSION}

Proposed system focus on different ways of operating and controlling electrical and electronic appliances remotely which are intern connected to Arduino which acts as intermediate between android application and signals from devices. This method of controlling such applications is referred to as automation. Our method presents the automated approach of controlling the household devices that could ease the tasks of using the traditional methods The system is fully functional and operational through android application. Added speech recognition to the system reduces their dependency on the other people to carry out day to day activities. Application has braille identifier, which help disabled people to not to depend on others. This system is not only helpful for disabled people but also provides opportunity for common people. 


\section{Virtual Assistant App for Disabled People}

\section{REFERENCES}

1. Amrita S. Tulshan and Sudhir NamdeoraoDhage, Survey on Virtual Assistant: Google Assistant, Siri, Cortana, Alexa, ${ }^{\text {th }}$ International Symposium SIRS 2018, Bangalore, India, 2018,pno 191-200.

2. Avni Sharma, Rinkesh Patel, Home Automation Using Augmented Reality Vol 1 Jan-June 2017, International Journal Of Electricals and

3. Electronics Engineering.

4. Md. Siddiqur Rahman Tanveer, M.M.A. Hashem and Md. Kowsar Hossain, Android Assistant EyeMate for Blind and Blind Tracker, Procs. of the IEEE 2015 18th International Conference on Computer and Information Technology (ICCIT 2015), Dhaka, Bangladesh, pno 266-271.

5. S. Supriya , A. Senthilkumar, Electronic Braille pad, 4-6 June 2009 International Conference on Control, Automation, Communication and Energy Conservation

6. Li Nian-feng ; Wang Li-rong, A kind of Braille paper automatic marking system, 19-22 Aug. 2011, 2011 International Conference on Mechatronic Science, Electric Engineering and Computer (MEC)

7. Sung Jae Kang, Young Ho, Kim, In Hyuk Moon, "Development Of An Intelligent Guide-Stick For

8. The Blind", IEEE International Conference on Robotics \& Automation Seoul, Korea, May 21-26, 2001.

9. Johann Borenstein and Iwan Ulrich, "The Guide Cane- A Computerized Travel Aid for The Active

10. Guidance OfBlindPedestrians", IEEE International Conference on Robotics and Automation, Albuquerque, NM, Apr. 21-27, 1997.

11. Zhang, Shanjun, and Kazuyoshi Yoshino. "A braille recognition system by themobile phone with embedded camera." In Innovative Computing, Information and Control, 2007.

12. ICICIC'07. Second International Conference on, pp. 223-223. IEEE, 2007.

\section{AUTHORS PROFILE}

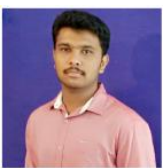

Mr. Nithin Gowda N. S., Department of CSE, Malnad College of Engineering, Hassan. His research interests are Cloud Computing and Network Secuirty

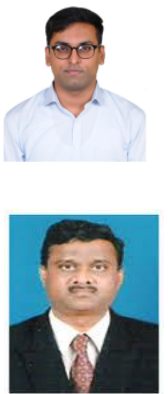

Mr. Yashas Gowda H. M., Department of CSE, Malnad College of Engineering, Hassan. His research interests are Cloud Computing and Network Secuirty.

Dr. Ramesh B. is currently working asProfessor in Department of CSE, Malnad College of Engineering, Hassan. He has published more than 50 research papers in prominent National and International Journals. His research areas are Computer Networks, Multimedia Computing, Mobile AdHoc Networks, Cloud Computing and Network Secuirty 